\title{
Dispersive analysis of light-meson transition form factors
}

\author{
Bastian Kubis ${ }^{1, \star}$ \\ ${ }^{1}$ Helmholtz-Institut für Strahlen- und Kernphysik (Theorie) and Bethe Center for Theoretical Physics, \\ Universität Bonn, 53115 Bonn, Germany
}

\begin{abstract}
We discuss status and prospects of a dispersive analysis of the $\pi^{0}, \eta$, and $\eta^{\prime}$ transition form factors. Particular focus is put on the various pieces of experimental information that serve as input to such a calculation. These can help improve on the precision of an evaluation of the light pseudoscalar pole contributions to hadronic lightby-light scattering in the anomalous magnetic moment of the muon. ${ }^{\star \star}$
\end{abstract}

\section{Hadronic light-by-light scattering and the anomalous magnetic moment of the muon}

The uncertainty of the Standard-Model prediction of the anomalous magnetic moment of the muon is entirely dominated by hadronic contributions [2]. While the dominant hadronic vacuum polarization can be expressed, via a dispersion relation, in terms of measurable $e^{+} e^{-} \rightarrow$ hadrons total cross sections, the situation for the $\alpha_{\mathrm{QED}}$-suppressed hadronic light-by-light scattering is less straightforward.

This presentation is part of an effort to analyze also the hadronic light-by-light scattering tensor using dispersion theory [3, 4]. Dispersion theory makes maximal use of analyticity and unitarity, two fundamental consequences of relativistic quantum field theories that mathematically incorporate the principles of causality and probability conservation. The various (in principle infinitely many different) contributions to hadronic light-by-light scattering are organized in terms of their analytic structure, according to the cuts and poles in the different energy variables that are dictated by the above principles. This has the advantage that all contributions will be given in terms of on-shell form factors and scattering amplitudes, hence observables that can to a large extent again be related to experimentally accessible quantities [5]. An ordering principle will be to consider intermediate states in terms of increasing masses, hence the lightest pseudoscalar pole terms $\left(\pi^{0}, \eta, \eta^{\prime}\right)$ as well as pion-pion intermediate states are expected to give the largest individual contributions. In addition, all such form factors and scattering amplitudes can in turn also be analyzed using dispersion theory: they are reconstructed from their discontinuities or imaginary parts, whose most important contributions are determined by the leading hadronic intermediate states. One therefore interrelates a larger set of possible data, and often can avoid the direct use of amplitudes with leptons in the final state that are strongly suppressed by powers of the fine structure constant $\alpha_{\mathrm{QED}}$. We will here concentrate on the $\pi^{0}, \eta$, and $\eta^{\prime}$ transition form factors that determine the corresponding pole terms; parallel efforts exist for the pion loop including rescattering effects $[6,7]$.

\footnotetext{
^e-mail: kubis@hiskp.uni-bonn.de

${ }^{\star}$ These proceedings borrow heavily from a previous conference contribution [1].
} 


\section{Dispersion relation for $\pi^{0}, \eta, \eta^{\prime}$ singly-virtual transition form factors}

\subsection{Intermediate states}

In order to write down dispersion relations for the transition form factors $F_{P \gamma^{*} \gamma^{*}}\left(q_{1}^{2}, q_{2}^{2}\right)$ that describe the processes $P \rightarrow \gamma^{*} \gamma^{*}, P=\pi^{0}, \eta, \eta^{\prime}$, we first decompose these in terms of isospin: while the neutral pion always needs to decay into one isovector and one isoscalar photon $(v s)$, the $\eta^{(\prime)}$ decay is given by the sum of an isovector-isovector $(v v)$ and an isoscalar-isoscalar $(s s)$ contribution,

$$
F_{\pi^{0} \gamma^{*} \gamma^{*}}\left(q_{1}^{2}, q_{2}^{2}\right)=F_{v s}\left(q_{1}^{2}, q_{2}^{2}\right)+F_{v s}\left(q_{2}^{2}, q_{1}^{2}\right), \quad F_{\eta^{(\prime)} \gamma^{*} \gamma^{*}}\left(q_{1}^{2}, q_{2}^{2}\right)=F_{v v}\left(q_{1}^{2}, q_{2}^{2}\right)+F_{s s}\left(q_{1}^{2}, q_{2}^{2}\right) .
$$

We need to identify the dominant intermediate states at low energies, where high precision is of paramount importance. For the isovector photons, the most important intermediate state consists of a pair of charged pions, while for the isoscalar ones, three pions are the lightest option. The isovector dispersion relation will then express the transition form factor in terms of the product of the pion vector form factor $F_{\pi}^{V}(s)$ and the amplitude $\gamma^{(*)} \pi^{0} \rightarrow \pi^{+} \pi^{-}$in the case of the $\pi^{0} /$ the decay amplitude(s) $\eta^{(\prime)} \rightarrow \pi^{+} \pi^{-} \gamma^{(*)}$ for the $\eta^{(\prime)}$. The most general description of the three-pion intermediate state for the isoscalar photons, in contrast, would be a lot more complicated, and certain approximations at least for the transitions $3 \pi \rightarrow \gamma^{(*)} \pi^{0} / \eta^{(\prime)}$ are almost unavoidable [8]. Fortunately, the vector-isoscalar spectral function at low energies is strongly dominated by the narrow $\omega$ and $\phi$ resonances, such that a vector-meson-dominance approximation is justified here to a large extent.

\subsection{Universality of final-state interactions, $\eta^{(\prime)} \rightarrow \pi^{+} \pi^{-} \gamma$}

Final-state interactions between two strongly interacting particles can be described in terms of form factors, which can be linked to the properties of scattering amplitudes using analyticity and unitarity. The unitarity relation for a pion form factor $F_{J}^{I}(s)$ of isospin $I$ and angular momentum $J$ reads

$$
\operatorname{disc} F_{J}^{I}(s)=2 i \operatorname{Im} F_{J}^{I}(s)=2 i F_{J}^{I}(s) \times \theta\left(s-4 M_{\pi}^{2}\right) \times \sin \delta_{J}^{I}(s) e^{-i \delta_{J}^{I}(s)},
$$

from which one deduces Watson's theorem: the form factor shares the phase $\delta_{J}^{I}(s)$ of the (elastic) scattering amplitude. The solution to Eq. (2) is obtained in terms of the Omnès function $\Omega_{J}^{I}(s)$,

$$
F_{J}^{I}(s)=P_{J}^{I}(s) \Omega_{J}^{I}(s), \quad \Omega_{J}^{I}(s)=\exp \left\{\frac{s}{\pi} \int_{4 M_{\pi}^{2}}^{\infty} \mathrm{d} x \frac{\delta_{J}^{I}(x)}{x(x-s)}\right\},
$$

where $P_{J}^{I}(s)$ is a polynomial that needs to be determined by other methods, e.g. by matching to chiral perturbation theory. The pion vector form factor $F_{\pi}^{V}(s)$ as extracted from $\tau^{-} \rightarrow \pi^{-} \pi^{0} v_{\tau}$ decays, e.g., can be described very accurately by a representation (3) up to $\sqrt{s}=1 \mathrm{GeV}$, employing a linear polynomial $R(s) \equiv P_{1}^{1}(s)=1+\alpha_{V} s$-at higher energies, the nonlinear effects of inelastic resonances become important [9]. The slope parameter $\alpha_{V}$ therein is relatively small, $\alpha_{V} \sim 0.1 \mathrm{GeV}^{-2}$.

The power of the universality of final-state interactions lies in the fact that an Omnès representation (3) will apply everywhere where two pions are produced from a point source in a relative $P$-wave; the process-dependence can be reduced to the coefficients of the multiplicative polynomial. Such a representation can in particular be used for the decays $\eta^{(\prime)} \rightarrow \pi^{+} \pi^{-} \gamma$ [10]: they are driven by the chiral anomaly and require the pion pair to be in an odd partial wave, hence the assumption of dominance by the $P$-wave $f_{1}(s)$ is entirely justified. For the decay of the $\eta$, an ansatz with a linear polynomial, $f_{1}(s)=A(1+\alpha s) \Omega_{1}^{1}(s)$, was shown to be sufficient to describe the data in the physical decay region, however with a large slope: $\alpha \sim 1.5 \mathrm{GeV}^{-2}$ [11-13]. This is somewhat remarkable: while $\alpha_{V}$ can 

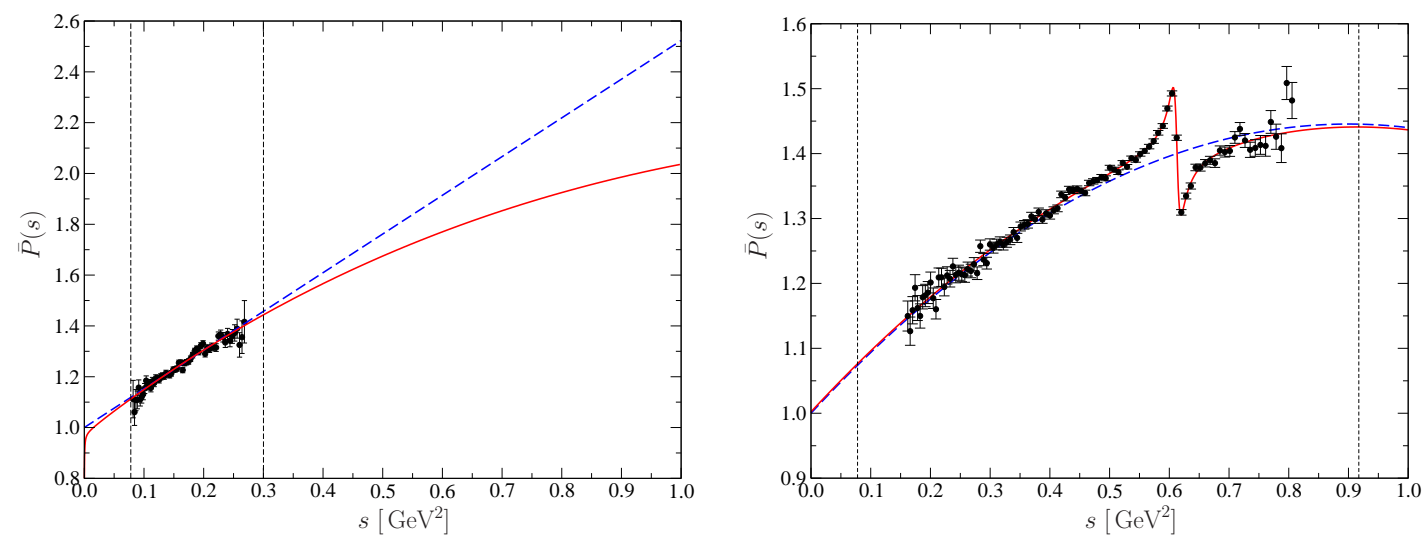

Figure 1. Left panel: representation of the decay distribution $\eta \rightarrow \pi^{+} \pi^{-} \gamma$ [12] to allow for comparison to the (normalized) polynomial $\bar{P}(s)=1+\alpha s$ (blue dashed curve). The full red curve includes the effects of $a_{2}$ exchange in addition. Right panel: the same distribution for $\eta^{\prime} \rightarrow \pi^{+} \pi^{-} \gamma$ [14], showing the theoretical fit of Ref. [15] without (blue dashed) and with (red) effects of $\rho-\omega$ mixing. The vertical dashed lines in both cases denote the boundaries of phase space at $s=4 M_{\pi}^{2}$ and $s=M_{\eta^{(\prime)}}^{2}$.

be understood as the low-energy tail of higher resonances in the region $1 \mathrm{GeV} \lesssim \sqrt{s} \lesssim 2 \mathrm{GeV}$, such an interpretation seems implausible for the $\eta \rightarrow \pi^{+} \pi^{-} \gamma$ decay. Given that the dispersion integral to calculate the transition form factor formally extends to infinity, deviations from the linear rise in $P(s)$ may become important. In order to judge how reliable such an approximation is, we may consider the possible effects of nontrivial $\pi \eta$ dynamics-so-called left-hand cuts or crossed-channel effects, something that has been neglected so far. The lowest-lying resonance to contribute in $\eta \rightarrow \pi^{+} \pi^{-} \gamma$ turns out to be the $D$-wave $a_{2}$ (1320) [13]. The result of a dispersive solution including the effects of $a_{2}$-exchange is shown in Fig. 1(left): the left-hand cut induces a curvature in $P(s)$ that has only very small effects in the decay region; however, if one evaluates a sum rule for the slope of the form factor at $q^{2}=0$, one finds that the $a_{2}$ effects reduce this slope by $7-8 \%$.

The same reasoning can equally be applied to an analysis of the decay $\eta^{\prime} \rightarrow \pi^{+} \pi^{-} \gamma$, for which a new measurement by BESIII [14] is even more conclusive than the $\eta$ decay data, as the larger phase space of the $\eta^{\prime}$ decay allows one to see deviations from a linear polynomial much more clearly. The data demonstrate the need of a quadratic term therein to very high significance; moreover, they are so precise that even the isospin-breaking $\rho$ - $\omega$-mixing effect is clearly discernible [15]. The fit to pseudo-data generated according to preliminary results is shown in Fig. 1(right).

\subsection{Dispersive prediction for $\eta^{(\prime)} \rightarrow \ell^{+} \ell^{-} \gamma$}

With the input to the isovector dispersion relation fixed, we can calculate the singly-virtual $\eta^{(\prime)}$ transition form factors. The resulting prediction includes the propagated uncertainties both from the experimental input employed and due to the high-energy continuation of the dispersion integral [9]. It can be compared to the experimental data on the decays $\eta^{(\prime)} \rightarrow \ell^{+} \ell^{-} \gamma$ [16-20]. In Fig. 2, we observe one of the main strengths of the dispersive approach: by fixing the input to the singly-radiative decays $\eta^{(\prime)} \rightarrow \pi^{+} \pi^{-} \gamma$ (with the decay rate scaling according to $\propto \alpha_{\mathrm{QED}}$ ), we gain a huge statistical advantage over the direct measurements of $\eta^{(\prime)} \rightarrow \ell^{+} \ell^{-} \gamma$ (rate $\left.\propto \alpha_{\mathrm{QED}}^{3}\right)$. A direct measurement of the transition form factors of comparable precision to the theoretical calculation based on dispersion 

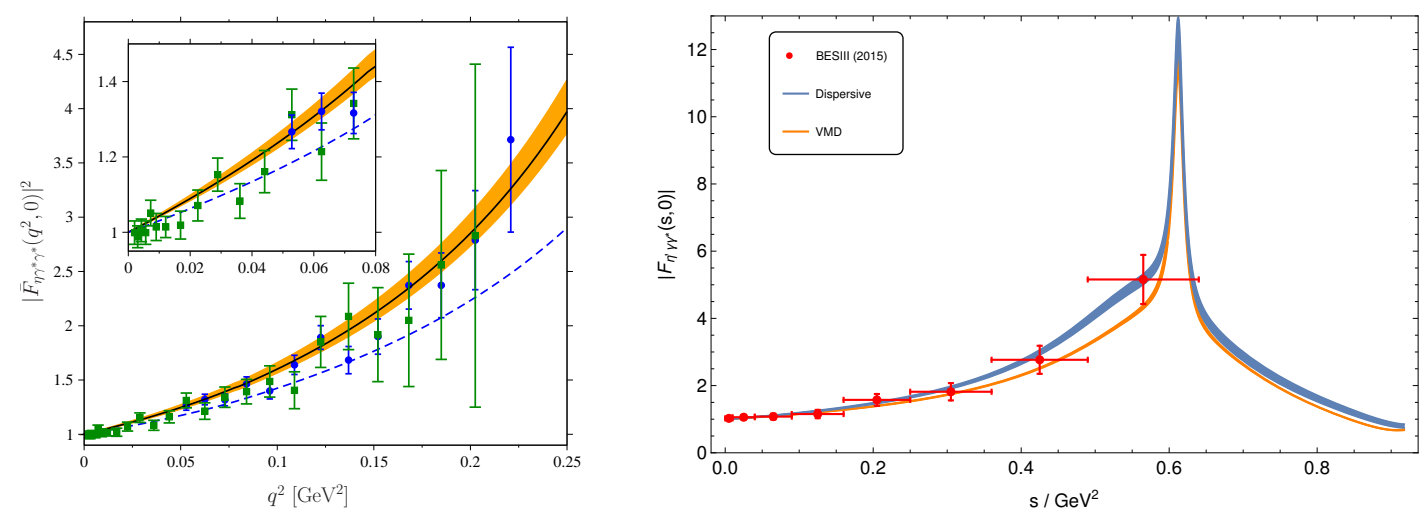

Figure 2. Left panel: Comparison of the dispersive prediction for $\left|\bar{F}_{\eta \gamma^{*} \gamma^{*}}\left(q^{2}, 0\right)\right|^{2}$ to data from the A2 [16] (green) and NA60 [19] (blue) collaborations. Figure courtesy of C. Hanhart. Right panel: Dispersion-theoretical prediction for the singly-virtual $\eta^{\prime}$ transition form factor (blue band), compared to a vector-meson-dominance model (orange), as well as the data points taken by the BESIII collaboration [20]. Figure courtesy of S. Holz [21].

relations will be enormously difficult. One observes in Fig. 2(right) that the isoscalar contribution for the $\eta^{\prime}$ is larger; the narrow $\omega$ peak on top of the broad resonance shape due to the $\rho$ enhancement is clearly visible. However, it can be modeled well using the vector-meson-dominance assumption, with the necessary coupling constants extracted directly from the partial decay widths for $\eta^{\prime} \rightarrow \omega \gamma$ and $\omega \rightarrow \ell^{+} \ell^{-}$as well as $\phi \rightarrow \eta^{\prime} \gamma$ and $\phi \rightarrow \ell^{+} \ell^{-}$.

\subsection{Dispersive analysis of the $\pi^{0}$ transition form factor}

As outlined in Sec. 2.1, the process $\gamma \pi \rightarrow \pi \pi$ plays the analogous role for the $\pi^{0}$ transition form factor that $\eta^{(\prime)} \rightarrow \pi^{+} \pi^{-} \gamma$ has for the $\eta^{(\prime)}$. A dispersive description of $\gamma \pi \rightarrow \pi \pi$ is discussed in Ref. [22]; due to full crossing symmetry, it is even more restrictive and can be solved using Khuri-Treiman techniques. In addition to serving as an input to the transition form factor analysis, the dispersive representation can also be used to extract the chiral anomaly, which determines the amplitude at zero energy (and quark mass), from the upcoming high-precision experimental data of the COMPASS collaboration [23].

The generalization of this amplitude to virtual photons is intimately related to the isoscalar-vectormeson decay amplitudes $\omega / \phi \rightarrow 3 \pi$ [24], which can be constructed similarly, taking into account certain complications in the analytic structure due to the decay kinematics. The resulting predictions of the corresponding Dalitz plot distributions have been compared favorably to the high-statistics $\phi \rightarrow 3 \pi$ Dalitz plot measured by the KLOE collaboration [25], while the first Dalitz plot data for $\omega \rightarrow 3 \pi$ by WASA-at-COSY are not yet conclusive as far as the subtle, non-trivial rescattering effects beyond two-body interactions are concerned [26].

Starting from the $3 \pi$ decay amplitudes, predictions for the vector-meson transition form factors $\omega \rightarrow \pi^{0} \gamma^{*}$ and $\phi \rightarrow \pi^{0} \gamma^{*}$ have been calculated dispersively [27, 28] (see also Ref. [29] for an extension to $J / \psi \rightarrow \pi^{0} \gamma^{*}$ ), which, despite a significant enhancement beyond the naive vector-meson-dominance picture, cannot explain the steep rise in the $\omega \rightarrow \pi^{0} \mu^{+} \mu^{-}$spectra measured in heavy-ion reactions by NA60 [18, 19], while more recent measurements of $\omega \rightarrow \pi^{0} e^{+} e^{-}[17]$ and $\phi \rightarrow \pi^{0} e^{+} e^{-}$[30] show no such conflict (but are also less precise). The NA60 data may actually violate very general unitarity bounds $[31,32]$. 

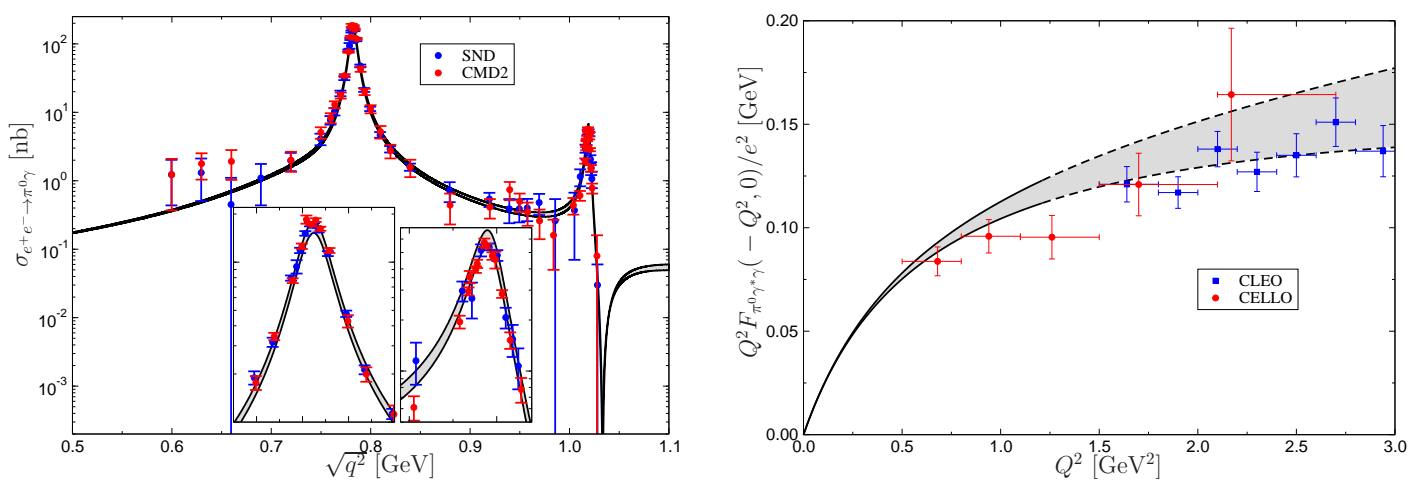

Figure 3. Left panel: $e^{+} e^{-} \rightarrow \pi^{0} \gamma$ cross section predicted from $e^{+} e^{-} \rightarrow 3 \pi$, compared to the data of Refs. [3335]. Right panel: Singly-virtual pion transition form factor in the space-like region, compared to CELLO [36] and CLEO [37] data. Figures taken from Ref. [8].

The full prediction for the $\pi^{0}$ transition form factor is obtained by generalizing the vector meson decays to an appropriate parametrization of the cross section $e^{+} e^{-} \rightarrow 3 \pi$, from which the singlyvirtual, timelike transition form factor as measured in $e^{+} e^{-} \rightarrow \pi^{0} \gamma$ can then be directly calculated [8]. The result agrees very well with the existing measurements, see Fig. 3(left). As the dispersive form factor representation naturally allows for analytic continuation, the spacelike transition form factor is easily obtained from the timelike one-the very precise prediction in particular at low spacelike momenta, see Fig. 3(right), the most relevant kinematic range for the muon's anomalous magnetic moment, still is to be tested by a forthcoming BESIII measurement.

\section{Outlook}

The efforts described above to obtain a model-independent, data-driven description of the $\pi^{0}, \eta$, and $\eta^{\prime}$ transition form factors is currently still being extended in various directions. In the case of the (dominant) $\pi^{0}$ pole contribution, efforts are underway to merge the dispersive low-energy description to the correct asymptotic high-energy behavior. For the $\eta$ and the $\eta^{\prime}$, more detailed investigations are necessary to understand the doubly-virtual transition form factors: attempts in this direction consider data on the reaction $e^{+} e^{-} \rightarrow \eta \pi^{+} \pi^{-}[38,39]$, as well as building a double spectral function from the purely hadronic decay amplitude $\eta^{\prime} \rightarrow \pi^{+} \pi^{-} \pi^{+} \pi^{-}$[40, 41]. The combination of further theoretical development along the lines sketched above, and improved experimental input for such a data-driven approach will therefore pave the way to a determination of the pseudoscalar pole contributions to hadronic light-by-light scattering with controlled uncertainties.

Acknowledgements I would like to thank the organizers of the PhiPsil7 for the invitation to a wonderful workshop. This research is supported in part by the DFG through funds provided to the Sino-German CRC 110 "Symmetries and the Emergence of Structure in QCD."

\section{References}

[1] B. Kubis, proceedings contribution, in: KLOE-2 Workshop on $e^{+} e^{-}$collision physics at $1 \mathrm{GeV}$, to appear in EPJ Web Conf. 
[2] F. Jegerlehner and A. Nyffeler, Phys. Rept. 477, 1 (2009)

[3] G. Colangelo, M. Hoferichter, M. Procura and P. Stoffer, JHEP 1409, 091 (2014)

[4] G. Colangelo, M. Hoferichter, M. Procura and P. Stoffer, JHEP 1509, 074 (2015)

[5] G. Colangelo et al., Phys. Lett. B 738, 6 (2014)

[6] G. Colangelo, M. Hoferichter, M. Procura and P. Stoffer, Phys. Rev. Lett. 118, 232001 (2017)

[7] G. Colangelo, M. Hoferichter, M. Procura and P. Stoffer, JHEP 1704, 161 (2017)

[8] M. Hoferichter et al., Eur. Phys. J. C 74, 3180 (2014)

[9] C. Hanhart et al., Eur. Phys. J. C 73, 2668 (2013) [Erratum: Eur. Phys. J. C 75, 242 (2015)]

[10] F. Stollenwerk et al., Phys. Lett. B 707, 184 (2012)

[11] P. Adlarson et al. [WASA-at-COSY Collaboration], Phys. Lett. B 707, 243 (2012)

[12] D. Babusci et al. [KLOE Collaboration], Phys. Lett. B 718, 910 (2013)

[13] B. Kubis and J. Plenter, Eur. Phys. J. C 75, 283 (2015)

[14] S.-s. Fang [BESIII Collaboration], PoS CD 15, 032 (2016)

[15] C. Hanhart et al., Eur. Phys. J. C 77, 98 (2017)

[16] P. Aguar-Bartolomé et al. [A2 Collaboration], Phys. Rev. C 89, 044608 (2014)

[17] P. Adlarson et al., Phys. Rev. C 95, 035208 (2017)

[18] R. Arnaldi et al. [NA60 Collaboration], Phys. Lett. B 677, 260 (2009)

[19] R. Arnaldi et al. [NA60 Collaboration], Phys. Lett. B 757, 437 (2016)

[20] M. Ablikim et al. [BESIII Collaboration], Phys. Rev. D 92, 012001 (2015)

[21] S. Holz, Bachelor thesis (University of Bonn, 2016)

[22] M. Hoferichter, B. Kubis and D. Sakkas, Phys. Rev. D 86, 116009 (2012)

[23] J. Seyfried, Master thesis (TU München, 2017)

[24] F. Niecknig, B. Kubis and S. P. Schneider, Eur. Phys. J. C 72, 2014 (2012)

[25] A. Aloisio et al. [KLOE Collaboration], Phys. Lett. B 561, 55 (2003) [Erratum: Phys. Lett. B 609, 449 (2005)]

[26] P. Adlarson et al. [WASA-at-COSY Collaboration], Phys. Lett. B 770, 418 (2017)

[27] S. P. Schneider, B. Kubis and F. Niecknig, Phys. Rev. D 86, 054013 (2012)

[28] I. V. Danilkin et al., Phys. Rev. D 91, 094029 (2015)

[29] B. Kubis and F. Niecknig, Phys. Rev. D 91, 036004 (2015)

[30] A. Anastasi et al. [KLOE-2 Collaboration], Phys. Lett. B 757, 362 (2016)

[31] B. Ananthanarayan, I. Caprini and B. Kubis, Eur. Phys. J. C 74, 3209 (2014)

[32] I. Caprini, Phys. Rev. D 92, 014014 (2015)

[33] M. N. Achasov et al., Eur. Phys. J. C 12, 25 (2000)

[34] M. N. Achasov et al., Phys. Lett. B 559, 171 (2003)

[35] R. R. Akhmetshin et al. [CMD2 Collaboration], Phys. Lett. B 605, 26 (2005)

[36] H. J. Behrend et al. [CELLO Collaboration], Z. Phys. C 49, 401 (1991)

[37] J. Gronberg et al. [CLEO Collaboration], Phys. Rev. D 57, 33 (1998)

[38] B. Aubert et al. [BaBar Collaboration], Phys. Rev. D 76, 092005 (2007) [Erratum: Phys. Rev. D 77, $119902(2008)]$

[39] C. W. Xiao et al., arXiv:1509.02194 [hep-ph]

[40] F.-K. Guo, B. Kubis and A. Wirzba, Phys. Rev. D 85, 014014 (2012)

[41] M. Ablikim et al. [BESIII Collaboration], Phys. Rev. Lett. 112, 251801 (2014) [Addendum: Phys. Rev. Lett. 113, 039903 (2014)] 